\title{
Measurement of lacunar bone strains and crack formation during tensile loading by digital volume correlation of second harmonic generation images
}

\author{
Scott Wentzell ${ }^{\mathrm{a}}$, Robert Sterling Nesbit ${ }^{\mathrm{a}, \mathrm{b}}$, James Macione ${ }^{\mathrm{a}, \mathrm{b}}$, Shiva Kotha ${ }^{\mathrm{a}, \mathrm{b}}$ \\ ${ }^{a}$ Rensselaer Polytechnic Institute, Troy, NY; ${ }^{b}$ University of Connecticut, Storrs-Mansfield, CT
}

\section{Corresponding Author:}

Scott Wentzell

Address:

CBIS 3131

$1108^{\text {th }}$ Street

Troy, NY, 12180

Email:

scott.a.wentzell@gmail.com

Phone:

862-266-5037 


\begin{abstract}
The maintenance of healthy bone tissue depends upon the ability of osteocytes to respond to mechanical cues on the cellular level. The combination of digital volume correlation and second harmonic generation microscopy offers the opportunity to investigate the mechanical microenvironment of intact bone on the scale of individual osteocytes. Adult human femurs were imaged under tensile loads of 5 and $15 \mathrm{MPa}$ and volumes of approximately 492x429x31 $\mu^{3}$ were analyzed, along with an image of a bone microcrack under the same loading conditions. Principal strains were significantly higher in three-dimensional digital volume correlation when compared to two-dimensional digital image correlation. The average maximum principal strain magnitude was 5.06-fold greater than the applied global strain, with peak strains of up to 23.14-fold over global strains measured at the borders of osteocyte lacunae. Finally, a microcrack that initiated at an osteocyte lacunae had its greatest tensile strain magnitudes at the crack expansion front in the direction of a second lacunae, but strain at the crack border was reduced to background strain magnitudes upon breaching the second lacunae. This serveed to demonstrate the role of lacunae in initiating, mediating and terminating microcrack growth.
\end{abstract}

Keywords: Second Harmonic Generation, Digital Volume Correlation, Osteocyte Lacunar Strain, Microcrack Strain

\title{
1. Introduction
}

As the average age of the population of the United States has advanced, concern about societal bone health has grown as well with fracture prevention and treatment a major cost in the United States health care system [1]. It has become clear through several decades of research that maintenance of bone strength depends upon bone remodeling mediated by mechanosensitive cell populations. Bone is a multihierarchical material, with physiological stresses translated from organ level domains to tissue and cellscale material features [2], [3]. Since bone cells, in particular osteocytes, are the primary candidates for mechanotransduction of physiological stresses, an understanding of the bone mechanical microenvironment has become paramount to elucidating osteocyte sensing mechanisms [4], [5]. Of particular interest is the measurement of strain concentration around osteocyte lacunae, as osteocytes have been shown to have osteogenic responses to strain but only at levels greater than physiological averages on the organ level [6], [7]. Bone remodeling has also been linked to microdamage, or material failure in the form of cracks on the micrometer scale [8]-[10]. The investigation of both of these phenomenon would help further describe the relationship between physiological loading and the mechanical environment of osteocytes.

Several methods have been developed previously to measure micrometer scale strains in bone tissue. The majority of these methods feature a combination of time course imaging before and after mechanical loading and digital calculation of the material strain fields from the resultant images [11][13]. A common and powerful technique for the measurement of strain fields from images is digital image correlation (DIC). In digital image correlation, images of the deformed and undeformed sample 
are compared. Through an iterative process the deformed image is transformed by variables describing the displacement and strain in the sample until the deformed image matches the original image as closely as possible. The matching process occurs in an iterative grid of image subsections, giving a matrix of displacements for the entire image of the sample [14]-[16]. Digital image correlation for strain measurement of bone has been reported for cortical bone surfaces as well as computed tomography data [11], [17], [18]. Two-dimensional DIC studies of osteocyte lacunae under stress have found strain amplification in the bone matrix at the osteocyte lacunae [17], [18]. DIC has also been used to show correlations between intracellular calcium and cellular strain for individual osteocytes [19]. However, expansion of these techniques to three dimensions (digital volume correlation or DVC) has been limited mainly to CT and micro-CT samples, as these allow imaging of intact bone specimens [20], [21].

The collagen matrix present in bone is also susceptible to second harmonic generation microscopy (SHGM) [22], [23]. Second harmonic generation utilizes the intrinsic ability of collagen to facilitate the generation of a single photon of doubled frequency from two incident photons [24], [25]. This intrinsic property of collagen allows its discrimination from other tissue components during imaging, and further allows three-dimensional imaging of collagen matrices [26]. Since collagen is the principal component of the bone organic matrix, SHGM is ideally suited to capturing images for digital volume correlation and bone strain analysis as it allows the analysis of intact bone volumes [27]. The current work presents the use of digital volume correlation of second harmonic generation microscopy images for the measurement of both lacunar strains and the strain behavior of microcracks in human cortical bone.

\section{Methods}

\subsection{Sample Loading}

Femurs from 60-70 year old male humans were purchased and processed into samples. Briefly, their ends were cut and the cortical mid-shafts shafts quartered using a bandsaw. Medial or lateral sections were then flattened to appropriate thickness using 80 grit sandpaper and polished with 120, 300 and 600 grit paper using a Buelher Ecomet 3 grinder cooled by a water jet. A Buehler Isomet 5000 saw was then used to cut individual samples with dimensions of $35 \times 1 \times 0.5 \mathrm{~mm}^{3}$. The sample ends were capped with a dental composite to enable gripping by the mechanical loader while preventing slippage or sample damage. Briefly, the sample ends were demineralized in $1 \mathrm{~N} \mathrm{HCl}$ for 30 seconds and then bound with 2-hydroxyethyl methacrylate (HEMA) via 30 seconds of ultraviolet exposure in the presence of camphorquinone and N-phenylglycine. The end caps were finished when bound to an acrylic sheet that contained a UV polymerized mix of bis-2-hydroxypropyl methacrylate (bisGMA), triethylene glycol dimethacrylate (TEGDMA), polymethyl methacrylate (PMMA), and the activators $1 \% \mathrm{w} / \mathrm{v}$ camphorquinone and $1 \% \mathrm{w} / \mathrm{w} \mathrm{N}$-phenylglycine).

\subsection{Sample Imaging and Loading}

A loading machine described previously was used to apply tensile loads to the human bone samples while being image by a Zeiss LSM 5 Multiphoton Confocal microscope in the backscattering configuration [27]. Imaging was performed with a Chroma D405/30 filter (Chroma Technology Corp, Bellows Falls, VT) at $800 \mathrm{~nm}$ wavelength excitation, which has been shown to induce second harmonic photon generation in collagen. Images were captured at room temperature via a water-immersion $\mathrm{W}$ Plan-Apochromat 20×/1.0 objective (Carl Zeiss Microscopy LLC, Thornwood, NY) with an image size of 
$2048 \times 2048$ pixels per XY plane and $0.82 \times 0.82 \mu \mathrm{m}^{2}$ resolution per pixel. The immersion connection between the lens and the bone sample was maintained by first soaking the sample in PBS, then by wrapping the exposed bone ends in PBS-saturated gauze. Three-dimensional Z-stacks of the XY images were composed of slices captured with 1.0 um spacing between slices, and the entire Z-stack comprising 50-100 slices. This gave a final voxel resolution of $0.82 \times 0.82 \times 1.0 \mu \mathrm{m}^{3}$. Tensile loads of 5 and $15 \mathrm{MPa}$ were applied to the samples to represent physiological loading levels, with waiting time added before imaging to account for sample creep during loading. Images were taken at each loading condition over approximately 30 minutes, and the global displacement of the sample measured by the encoder of the stepper motor with a resolution of approximately 1.2 micrometers.

\subsection{Digital Volume Correlation and Strain Calculation}

Pre-processing of the images was undertaken to increase the accuracy of the digital volume correlation. The intensity range of each image slice was normalized to the global maximum and minimum intensities of the Z-stack to mitigate the intensity loss with increased imaging depth. A Gaussian filter with a $3 \times 3 \times 3$ voxel kernel and sigma radius of 1 was then applied to the image volume to reduce errors in the sub-pixel displacement field when cubic interpolation is used. Finally, a Kalman stack filter was implemented across the Z-stack to reduce speckle noise across the image volume.

The displacement grid across the image volume was calculated by performing a montage of individual digital volume correlations. Correlations were performed at three different subvolumes of either $51 \times 51 \times 10,75 \times 75 \times 10$ or $101 \times 101 \times 10$ voxels for determination of the optimal analysis region size. For each image analysis region size, the montage of analysis had a step size of 20 voxels in the $\mathrm{X}$ and $\mathrm{Y}$ directions and 10 voxels in the $\mathrm{Z}$ direction. The analysis montage was iterated to form a grid of $31 \times 31 \times 3$ analysis points covering a total image volume area of $601 \times 601 \times 31$ voxels. The total analysis volume ranged from 30-70 micrometers below the surface of the bone sample. After calculation of the image volume displacement grid, the displacement values were corrected for sample rotations during loading which can pollute strain calculations. Lastly, the displacement grid was smoothed with a 3x3x1 median filter, analysis points that failed to converge were removed with a $5 \times 5 \times 3$ outlier filter, and the displacements tricubically interpolated across the $601 \times 601 \times 31$ total image analysis volume.

Strains were next calculated from the displacement grid. A three-dimensional Savitsky-Golay filter with a kernel of $5 \times 5 \times 5$ voxels was adapted from the literature which directly calculated the strain values [28]. Strain values measured along the $\mathrm{Z}$-axis $\left(\varepsilon_{\mathrm{ZZ}}, \varepsilon_{\mathrm{ZXZ}}, \varepsilon_{\mathrm{YZ}}\right)$ were all larger than predicted by the estimated Poisson's ratio by a factor of approximately 100. To reduce the influence of imaging effects on the strain measurements and allow comparison of the strain distributions, the displacement grids were aligned in the $\mathrm{Z}$-stack using the mean $\mathrm{X}$ and $\mathrm{Y}$ displacements of each individual slice. Further, the axial strains in the $\mathrm{Z}$ direction $\left(\varepsilon_{\mathrm{ZZ}}\right)$ were normalized to the range predicted by the Poisson's ratio of bone $(v=$ 0.3 ) by first fitting the strain distribution to a range of -1 to 1 and an average of 0 . The normalized distribution was then expanded over the range predicted by the planar (XY) strains. This preserved the distribution shape of the $\varepsilon_{Z Z}$ strains for comparison between samples while allowing calculation of the maximum and minimum principal strains.

\subsection{Statistical Analysis}


The average principle strains were first normalized to the global strain of each sample for statistical comparison. ANOVA and Tukey's Honest Significant Differences test were performed in R using the RStudio environment. For all statistical tests, a value of $\mathrm{p} \leq 0.05$ was considered significant.

\section{Results}

The effect of correlation region size on the measured strains was first examined. Average maximum and minimum principal strain magnitudes were not significantly changed as the correlation region area increased (Figure 1). Correlation regions below 51x51x11 voxels tended to not converge on a displacement solution, and so $51 \times 51 \times 11$ correlation regions were chosen for further investigation as they offered the highest resolution while producing viable displacement grids for analysis.

Displacement grids correlated to the direction of loading on the bone samples (Figure 2). The presence of image features like osteocyte lacunae matched changes in displacement magnitude and direction, with the greatest magnitude displacement vectors adjacent to lacunae. The magnitude and direction of the displacement vectors were also dependent upon the z-axis position of the analysis. The displacement vectors in regions of solid bone tended to closely match vectors of other XY planes, but differed near the edges of several lacunae. This implies that changing lacunar geometry in the z-axis was affecting displacements and therefore strains around lacunae.

Strains were calculated from the displacements grids using the image coordinate system, which was about 45 degrees off of the loading axis. A representative distribution of these axial strains ranged from approximately -0.03 to 0.03 (Figure 3).

Two-dimensional digital image correlation of second harmonic second images was previously reported. To investigate the difference in two-dimensional versus three-dimensional correlation, the principal strains were calculated for both analysis types for all samples, with a representative sample shown (Figure 4). Both the maximum and minimum principal strain magnitudes were significantly increased between the 2D and 3D image correlations. Maximum principal strains were on average 2.12 fold higher than the applied global strain for the $2 \mathrm{D}$ analysis, while the $3 \mathrm{D}$ analysis measured a 5.06 fold increase over applied strain. Likewise, 2D DIC measured minimum principal strains were -1.28 fold different applied strains, while 3D DVC minimum principal strains were -4.88 fold different.

Regions of greater magnitude maximum and minimum principal strains were co-located with the presence of osteocyte lacunae in the measured human bone samples (Figure 5). The average peak tensile principal strain among all three samples was 23.14 fold higher than the applied global strains, and average peak compressive principal strains was -27.48 fold greater than applied global strains. Further, all samples exhibited regions of pure compressive and pure tensile strain within the bone (negative maximum principal strain and positive minimum principal strain respectively). Localized increases in strain were also not seen unilaterally across all lacunae in a sample; there was no increase in strain around the edges of several lacunae across the bone samples. Figure 6 shows the three-dimensional distribution of strain in the sample and highlights lacunae with both increased and unaffected strain profiles. 
The image intensity of collagen fibrils under SHGM can change substantially with both fibril orientation and density, and fibril orientation and density are known to change in the immediate proximity of lacunae. Therefore, identification of the true edge of osteocyte lacunae is difficult in SHGM. To investigate differences in the strain environment between lamellar bone and the lacunae, the image volumes had a threshold applied to distinguish between 'collagen-bright' and 'collagen-dark' regions. Collagen-dark regions were expected to include the lacunae as well as a percentage of ossified bone tissue. The average principal strains in the bright and dark regions were compared (Figure 7). The magnitude of maximum and minimum principal strains was increased in collagen dark regions, but this difference was not significant.

During the strain analysis, a single sample with a microcrack between two lacunae was observed. Digital volume correlation was performed to obtain strain information at three locations: where the crack connected the two lacunae, at the crack leading edge, and the intact bone preceding the leading edge of the crack. The measured tensile strain was the key concern as it would lead to crack expansion (Figure 8). At the full crack location where each end of the crack was terminated in a lacuna, the maximum tensile strain was present at the outer edges of the lacunae away from the crack (Figure $8 \mathrm{~A} / \mathrm{B}$ ). At the crack leading edge, the crack is terminated on one end at a lacuna and the crack front is present in intact bone near the second lacuna. At this location, a region of increased strain was present at the leading edge of the crack between the crack and the unbreached lacuna (Figure 8C/D) indicating possible expansion towards the lacuna. Finally, in the intact bone preceding the crack front, increased strain is again present at the edges of the osteocyte lacunae (Figure $8 \mathrm{E} / \mathrm{F}$ ) but not in the area between them where further crack expansion could be expected. The three dimensional distribution of the compressive and tensile strains can be seen Figure 9, with the crack location indicated by the black or yellow ellipses.

\section{DISCUSSION}

The overall goal of this work was to validate the use of DVC with SHGM while investigating the effects of osteocyte lacunae in the bone matrix on the levels of strain in the collagen matrix at the cellular level. Three-dimensional analysis was shown to measure strains that were significantly higher than two-dimensional digital image correlation and highlights the importance of analyzing the entire bone volume. The average principal strain magnitude at this size order was approximately 5 times greater than the applied global strain, with up to 23 fold increases of strain at the edges of lacunae. The expansion of bone microcracks was also shown to be mediated by the presence of osteocyte lacunae, serving as both initiation points of cracks during stress concentration as well as blunting the further expansion of cracks when the breach lacunae.

\subsection{Validation of 3D DIC method}

Two-dimensional digital image correlation of bone has been demonstrated previously at bone surfaces as well as in three dimensional SHGM images. Three-dimensional digital volume correlation 
has been used in conjunction with datasets from computerized tomography, however this is the first demonstration three-dimensional digital volume correlation of SHGM images. In agreement with our previous work in two-dimensions, there exists a cutoff point where increasing the correlation area does not alter the magnitudes of principal strains measured (Figure 1) [27]. However, in three-dimensions small image volumes often fail to correlate, and so for the levels of contrast achieved with the presented SHGM images a minimum correlation area of $51 \times 51 \times 11$ was required. This corresponded to a volume of

$41 \times 41 \times 8.2 \mu \mathrm{m}^{3}$, which is on the approximate size order of individual lacunae. When iterated at this size order, displacement grids describing the lacunae strain microenvironment could be generated and analyzed (Figure 2). The benefit of performing measurements in three dimensions can be seen in the increased principal strains measured when compared to the previous two-dimensional analysis (Figure 4). The increase in measured maximum principal strain from 2-fold to 5-fold over global strains when moving to a three-dimensional analysis shows the importance of analyzing the entire strain microenvironment at this level.

\subsection{Strain Environment of Intact Bone}

In two dimensional surface digital image correlation by Nicollela et al., it was reported that strain values greater than 30,000 microstrain could be measured as a result of strain concentration at lacunae [18]. Concentration of strain at the site of lacunae has also been seen in finite element models [7]. This work offers further validation of increased strain due to lacunae, with maximum strain values up to 27 times the applied global strain measured (Figure 5). In the two-dimensional work by Nicolella et al. on bone milled to expose lacunae on the bone surface, the data showed strain magnitude increases of 2-7 times over the average background strain [18]. As the current measurements were made in a volume of bone that was fully intact and not subject to mechanical damage during sample preparation, this is an indication that strain values for in vivo bone tissue near osteocytes is likely at least an order of magnitude higher than global average strains. Osteocytes are the primary candidate of mechanotransduction for osteogenesis and exhibit osteogenic responses when subjected to strain [6], [29], [30]. However, it is also known physiological strain levels are not high enough to induce osteogenic responses in osteocytes. The amplification of strain by the lacunar structure in vivo may contribute to the ability of osteocytes to respond to physiological loads for bone maintenance. The demonstration that the three-dimensional strain environment of lacunae can magnify strains by an average of 5.06 times and a maximum of 23 times is therefore relevant to mechanotransduction mechanisms in vivo (Figure 4).

The three dimensional strain profiles also indicated that the osteocyte mechanical microenvironment was not uniform across all lacunae. The bone samples as a whole exhibited large variability (Figure 3), particularly near lacunae. For the representative sample in Figure 6, two lacunae experienced the highest tensile and compressive strains, while another lacunae did not have elevated strains. The possibility of stress shielding by surrounding lacunae has implications for the formation of microdamage in bone, as well as osteocyte mechanotransduction. The heterogeneous strain environment dictates where bone failure and resorption is most likely to occur.

\subsection{Mediation of Microcracks by Osteocyte Lacunae}


Microdamage has been seen to be initiated at osteocyte lacunae [31]. Microcracks have also been associated with bone resorption and bone resorption is known to be initiated at least partially in vivo by osteocytes [32], [33]. The existence of a crack in one of our samples offered the unique opportunity to investigate three-dimensional strain environment around a microcrack between to lacunae. Where the crack connected both lacunae, peak strain in the sample was at the outer edges of the lacunae. The sudden increase in crack radius upon reaching the second lacunae likely prevented further expansion by reducing stress concentration, which can be seen was entirely on the lacunae and not the crack (Figure 8 A/B). At the image depth of crack expansion, the crack appears to be initiated from one lacuna (Figure 8 $\mathrm{C} / \mathrm{D})$. The propagation front of the crack shows a region of increased tensile strain closest to the second lacunae, which implies the crack will open further towards the second lacuna. This indicates that lacunae also serve to direct microcrack expansion and prevent further spread of the microdamage. This conclusion is supported by the work of Christen et al., who also showed that microcracks began at lacunae but tended to terminate at other lacunae [21]. Nicolella et al. also found strain concentration at both the leading edge of a crack as well as at osteocyte lacunae, which lead to peak strains at these locations [17]. As the accumulation of microdamage is associated both with resorption activity and bone failure, an understanding of how microcrack expansion is mediated by the very structure of mechanosensitive osteocytes in bone merits further work.

\subsection{Limitations}

The proposed technique was able to generate displacement and strain values in a volume smaller than 500 micrometers across. However, the measured strains across the image depth were larger than predicted by a factor of approximately 100 but differing for each sample. These values altered the magnitude and direction of the principal strains towards the Z-axis. This was compensated for by normalizing the z-axis strains to the range predicted by the assumed isotropic Poisson's ratio calculated from XY planar strains of each unique bone sample. The average measured Poisson's ratio was approximately 0.3. While this did not allow for direct comparisons of the magnitude of z-axis strains developed in the sample, the shape of the distributions were still preserved. We have observed the dilation effect in imaged samples of bovine bone and mouse skin as well, which leads us to believe there is a change in the second harmonic generation behavior of the collagen under loading which merits further study to allow direct observations of the z-axis strains.

A further limitation lies in the need for access to extensive computing power. Three-dimensional analysis required large datasets to be calculated and analyzed in the process of digital volume correlation. Individual sample analysis times on robust desktop computers can be on the order of days, and were reduced by utilization of the supercomputing center present on the Rensselaer campus. However, access to this type of resource may be prohibitive.

\section{Conclusion}

The combination of digital image correlation with second harmonic generation microscopy allows for the analysis of the strain environment in bone on the cell level. Greater knowledge of the inhomogeneous nature of strain around osteocytes under various physiological loading conditions has key implications for the role and mechanisms of osteocytes as mechanotransducers. Further, the spatial interactions between osteocytes have been shown to affect crack propagation and stress shielding within 
bone volumes which merits further study on the role bone microgeometry plays on fatigue damage and bone remodeling.

\section{Acknowledgments}

We would like to acknowledge the contributions of Samantha Garrabrandt and Dr. Jean-Francois Dordick, both of whom provided technical assistance and insight. This work was supported by NSF/CMMI CAREER Grant \# 084869 and NIH/NIA Grant \# AG030637.

\section{Conflicts of Interest}

The authors have no conflicts of interest to report.

\section{References}

[1] Office of the Surgeon General (US), "Bone Health and Osteoporosis: A Report of the Surgeon General," Office of the Surgeon General (US), Rockville (MD), Book, 2004.

[2] D. Vashishth, "Hierarchy of bone microdamage at multiple length scales," Int. J. Fatigue, vol. 29, no. 6, pp. 1024-1033, Jun. 2007.

[3] J. Currey, "Measurement of the Mechanical Properties of Bone: A Recent History," Clin. Orthop. Relat. Res., vol. 467, no. 8, pp. 1948-1954, Mar. 2009.

[4] A. G. Robling et al., "Mechanical Stimulation of Bone in Vivo Reduces Osteocyte Expression of Sost/Sclerostin," J. Biol. Chem., vol. 283, no. 9, pp. 5866 -5875, Feb. 2008.

[5] J. Xiong and C. A. O'Brien, "Osteocyte RANKL: New insights into the control of bone remodeling," J. Bone Miner. Res., vol. 27, no. 3, pp. 499-505, Mar. 2012.

[6] Y. Han, S. C. Cowin, M. B. Schaffler, and S. Weinbaum, "Mechanotransduction and strain amplification in osteocyte cell processes," Proc. Natl. Acad. Sci. U. S. A., vol. 101, no. 47, pp. 16689-16694, Nov. 2004.

[7] A. Rath Bonivtch, L. F. Bonewald, and D. P. Nicolella, "Tissue strain amplification at the osteocyte lacuna: A microstructural finite element analysis," J. Biomech., vol. 40, no. 10, pp. 2199-2206, 2007.

[8] M. B. Schaffler, K. Choi, and C. Milgrom, "Aging and matrix microdamage accumulation in human compact bone," Bone, vol. 17, no. 6, pp. 521-525, Dec. 1995.

[9] D. Burr, "Microdamage and bone strength," Osteoporos. Int., vol. 14, no. 5, pp. 67-72, Sep. 2003.

[10] O. Verborgt, G. J. Gibson, and M. B. Schaffler, "Loss of Osteocyte Integrity in Association with Microdamage and Bone Remodeling After Fatigue In Vivo," J. Bone Miner. Res., vol. 15, no. 1, pp. 60-67, Jan. 2000.

[11] P. Sztefek et al., "Using digital image correlation to determine bone surface strains during loading and after adaptation of the mouse tibia," J. Biomech., vol. 43, no. 4, pp. 599-605, Mar. 2010.

[12] B. K. Bay, "Texture correlation: A method for the measurement of detailed strain distributions within trabecular bone," J. Orthop. Res., vol. 13, no. 2, pp. 258-267, Mar. 1995.

[13] E. Verhulp, B. va. Rietbergen, and R. Huiskes, "A three-dimensional digital image correlation technique for strain measurements in microstructures," J. Biomech., vol. 37, no. 9, pp. 1313-1320, Sep. 2004.

[14] T. C. Chu, W. F. Ranson, and M. A. Sutton, "Applications of digital-image-correlation techniques to," Exp. Mech., vol. 25, no. 3, pp. 232-244, Sep. 1985.

[15] W. H. Peters and W. F. Ranson, "Digital Imaging Techniques In Experimental Stress Analysis," Opt. Eng., vol. 21, no. 3, pp. 213427-213427-, 1982. 
[16] H. A. Bruck, S. R. McNeill, M. A. Sutton, and W. H. Peters, "Digital image correlation using Newton-Raphson method of partial differential correction,” Exp. Mech., vol. 29, pp. 261-267, Sep. 1989.

[17] D. P. Nicolella, A. E. Nicholls, J. Lankford, and D. T. Davy, "Machine vision photogrammetry: a technique for measurement of microstructural strain in cortical bone," J. Biomech., vol. 34, no. 1, pp. 135-139, Jan. 2001.

[18] D. P. Nicolella, D. E. Moravits, A. M. Gale, L. F. Bonewald, and J. Lankford, "Osteocyte lacunae tissue strain in cortical bone," J. Biomech., vol. 39, no. 9, pp. 1735-1743, 2006.

[19] A. L. Rath et al., "Correlation of cell strain in single osteocytes with intracellular calcium, but not intracellular nitric oxide, in response to fluid flow," J. Biomech., vol. 43, no. 8, pp. 1560-1564, May 2010.

[20] B. K. Bay, T. S. Smith, D. P. Fyhrie, and M. Saad, "Digital volume correlation: Three-dimensional strain mapping using X-ray tomography," Exp. Mech., vol. 39, no. 3, pp. 217-226, Sep. 1999.

[21] D. Christen et al., "Deformable image registration and 3D strain mapping for the quantitative assessment of cortical bone microdamage," J. Mech. Behav. Biomed. Mater., vol. 8, pp. 184-193, Apr. 2012.

[22] Y. Goulam Houssen, I. Gusachenko, M.-C. Schanne-Klein, and J.-M. Allain, "Monitoring micrometer-scale collagen organization in rat-tail tendon upon mechanical strain using second harmonic microscopy," J. Biomech., vol. 44, no. 11, pp. 2047-2052, Jul. 2011.

[23] R. Ambekar, M. Chittenden, I. Jasiuk, and K. C. Toussaint Jr., "Quantitative second-harmonic generation microscopy for imaging porcine cortical bone: Comparison to SEM and its potential to investigate age-related changes," Bone, vol. 50, no. 3, pp. 643-650, Mar. 2012.

[24] W. Mohler, A. C. Millard, and P. J. Campagnola, "Second harmonic generation imaging of endogenous structural proteins," Methods, vol. 29, no. 1, pp. 97-109, Jan. 2003.

[25] B. Kim, J. Eichler, K. M. Reiser, A. M. Rubenchik, and L. B. Da Silva, "Collagen structure and nonlinear susceptibility: Effects of heat, glycation, and enzymatic cleavage on second harmonic signal intensity," Lasers Surg. Med., vol. 27, no. 4, pp. 329-335, Jan. 2000.

[26] G. Cox et al., "3-Dimensional imaging of collagen using second harmonic generation," J. Struct. Biol., vol. 141, no. 1, pp. 53-62, Jan. 2003.

[27] S. Wentzell, R. Sterling Nesbitt, J. Macione, and S. Kotha, "Measuring strain using digital image correlation of second harmonic generation images," J. Biomech., vol. 46, no. 12, pp. 2032-2038, Aug. 2013.

[28] B. Pan, H. Xie, Z. Guo, and T. Hua, "Full-field strain measurement using a two-dimensional Savitzky-Golay digital differentiator in digital image correlation," Opt. Eng., vol. 46, no. 3, p. 033601, 2007.

[29] L. E. Lanyon, "Osteocytes, strain detection, bone modeling and remodeling," Calcif. Tissue Int., vol. 53, no. 1, pp. S102-S107, Feb. 1993.

[30] P. J. Ehrlich and L. E. Lanyon, "Mechanical Strain and Bone Cell Function: A Review," Osteoporos. Int., vol. 13, no. 9, pp. 688-700, Feb. 2014.

[31] G. C. Reilly, "Observations of microdamage around osteocyte lacunae in bone," J. Biomech., vol. 33, no. 9, pp. 1131-1134, Sep. 2000.

[32] T. C. Lee, A. Staines, and D. Taylor, "Bone adaptation to load: microdamage as a stimulus for bone remodelling," J. Anat., vol. 201, no. 6, pp. 437-446, Dec. 2002.

[33] S. Mori and D. B. Burr, "Increased intracortical remodeling following fatigue damage," Bone, vol. 14, no. 2, pp. 103-109, Mar. 1993. 



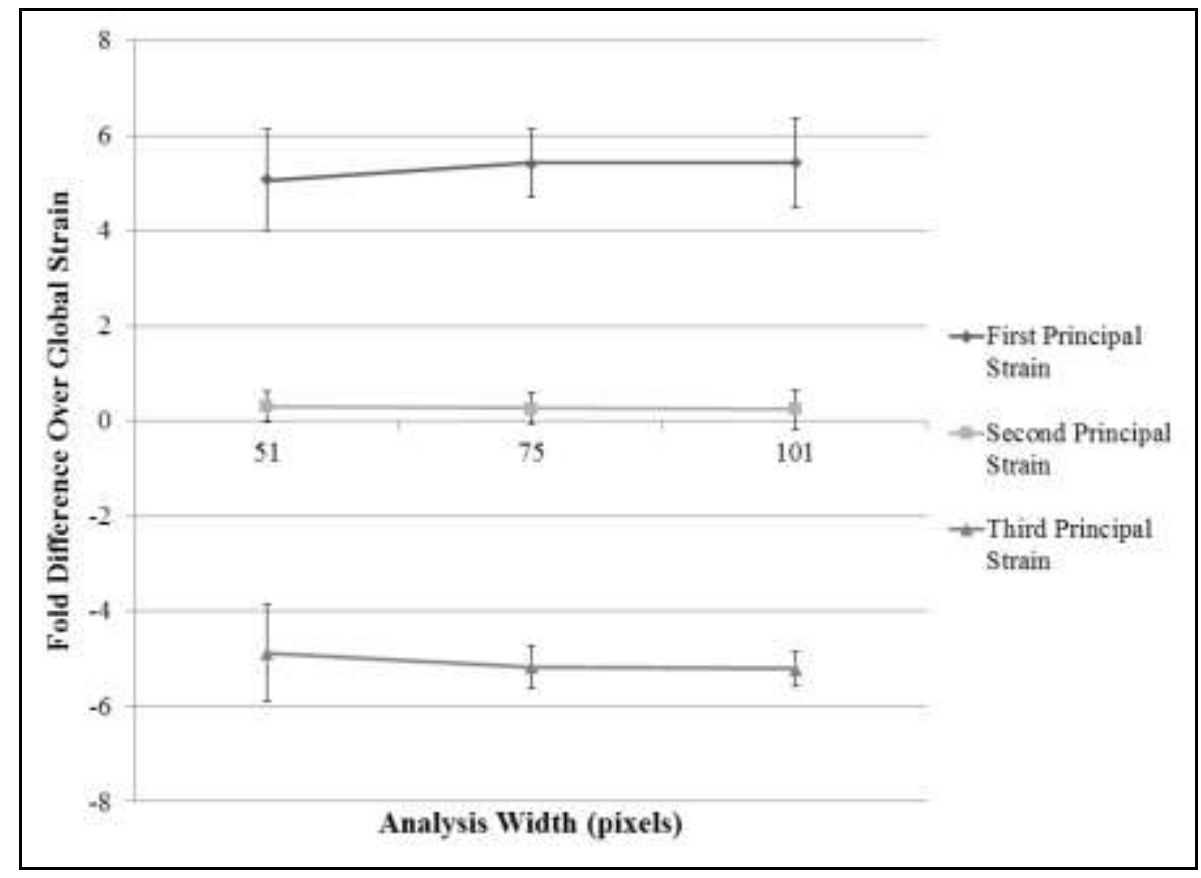

Figure 1. The effect of correlation region size on average principal strain magnitudes. Significant changes in average strain values were not seen with increasing correlation area. Due to this finding, the smallest correlation area (51x51x10 voxels) was used for strain analysis to maximize analysis resolution.

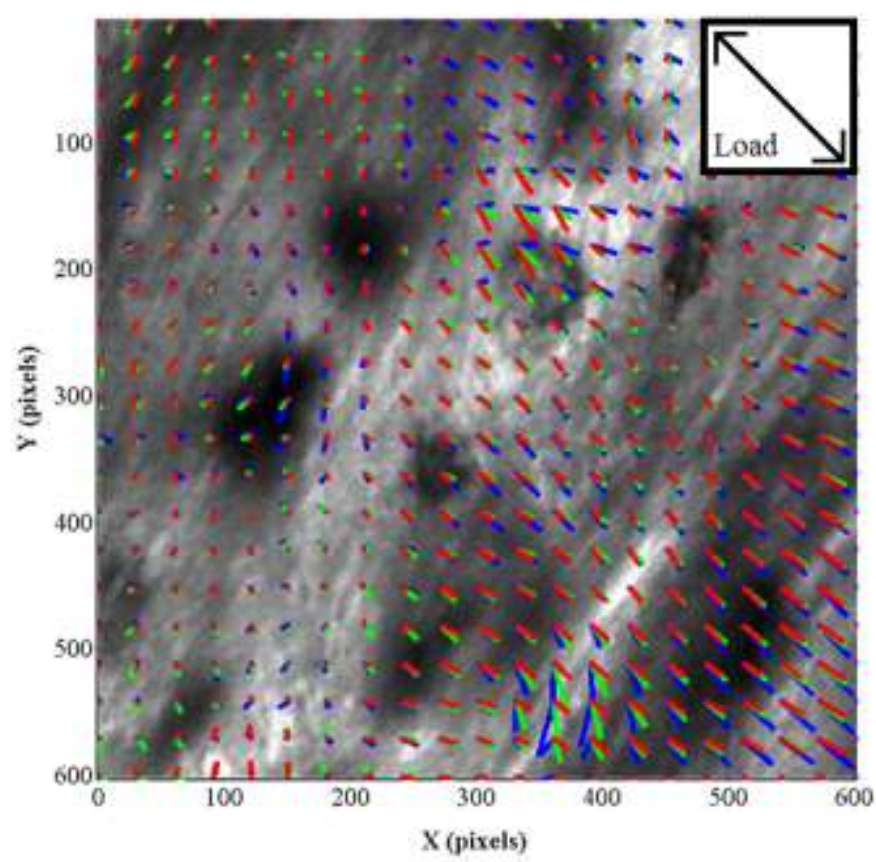


Figure 2. Representative displacement grid as measured by digital volume correlation. The applied loading was 45 degrees from the imaging coordinate system, and it can be seen that measured displacements followed this loading direction. The direction and magnitude of displacement vectors were also co-located with the presence of osteocyte lacunae. Three XY-planes of displacement are plotted, with red displacement vectors in the presented image plane, blue vectors 10 micrometers below and green vectors 20 micrometers below the image plane. A relationship between analysis depth and the displacement vectors can also be seen which is due to changing lacunar geometry in the z-axis.

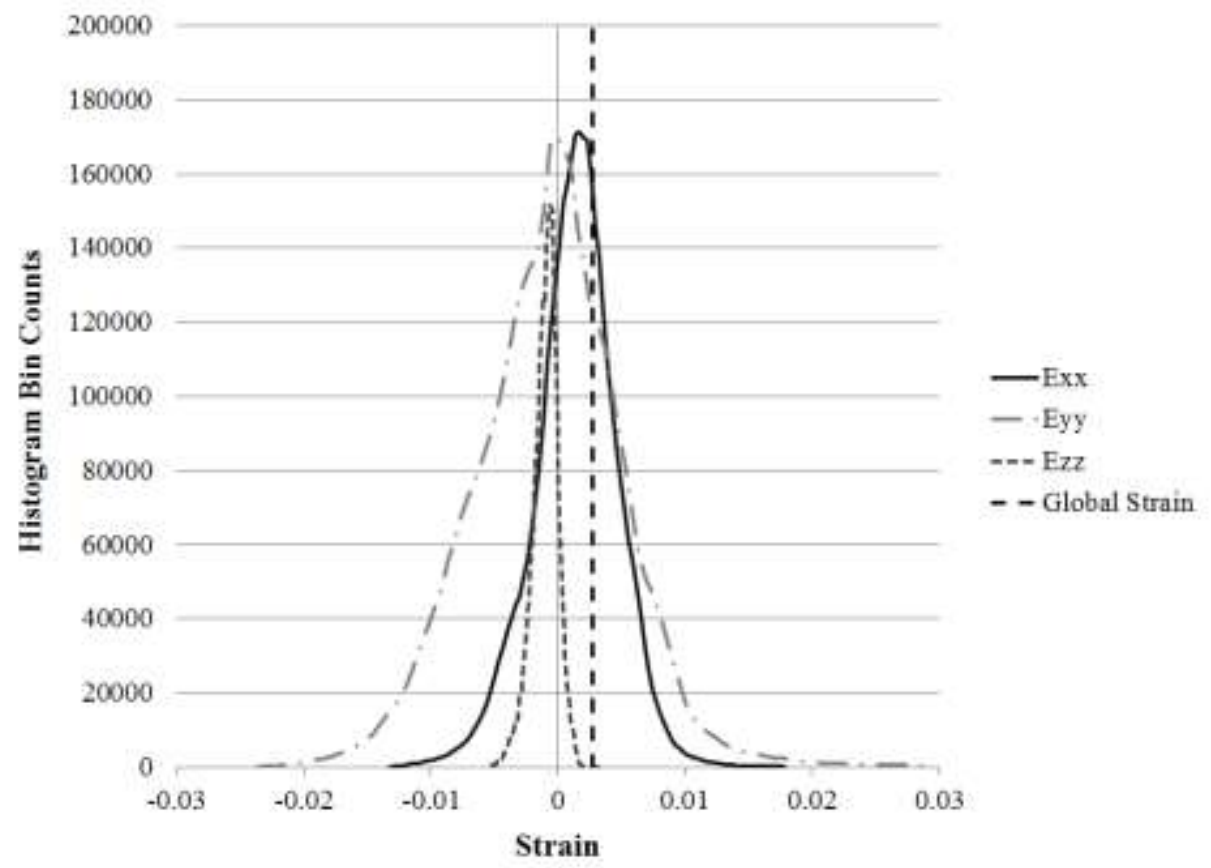

Figure 3. A representative axial strain distribution using the image coordinate system. The loading coordinate system was 45 degrees clockwise from the positive $x$-axis due to the configuration of the loading machine on the microscope stage. 


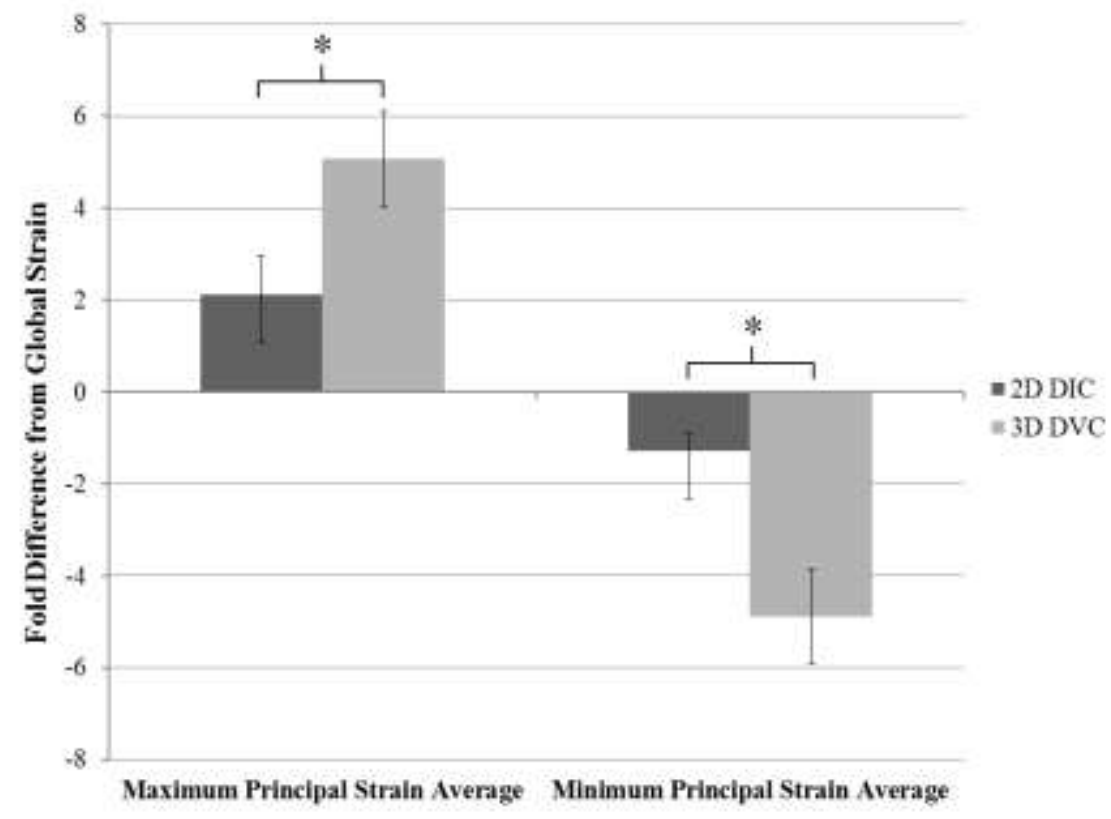

Figure 4. A comparison of average principal strains as calculated by 2-dimensional and 3-dimensional image correlation. The inclusion of $z$-axis strains in the analysis significantly increased the magnitudes of both maximum and minimum principal strain ( $p<0.05$ ). The 2-D principal strains were 2.12 and -1.28

fold the applied global strain, while 3-D principal strains were 5.06 and -4.88 fold the global strain.
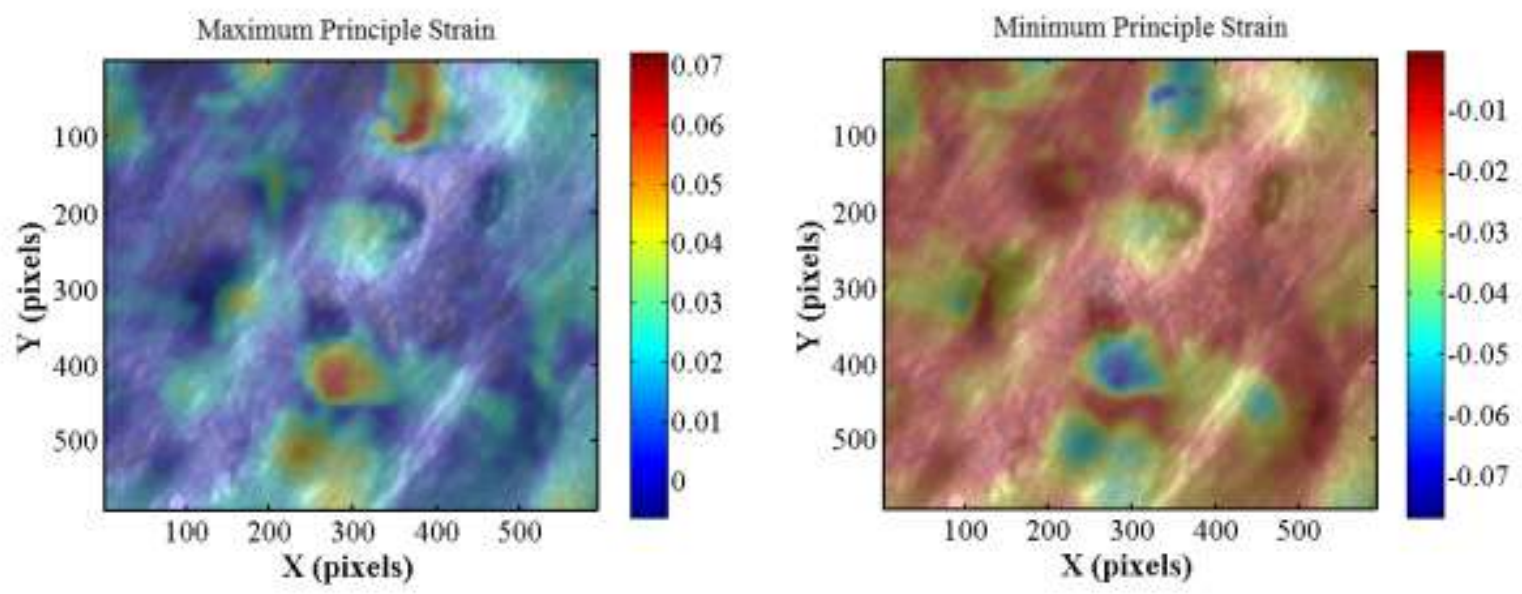

Figure 5. Maximum and minimum principal strains overlaid the bone SHGM images. The relative distribution of principle strain can be seen to correlate with the presence of osteocyte lacunae within the bone. However, several lacunae did not experience an increase in strain around their edges, indicating that the strain microenvironment is not uniform even for lacunae in close proximity. 

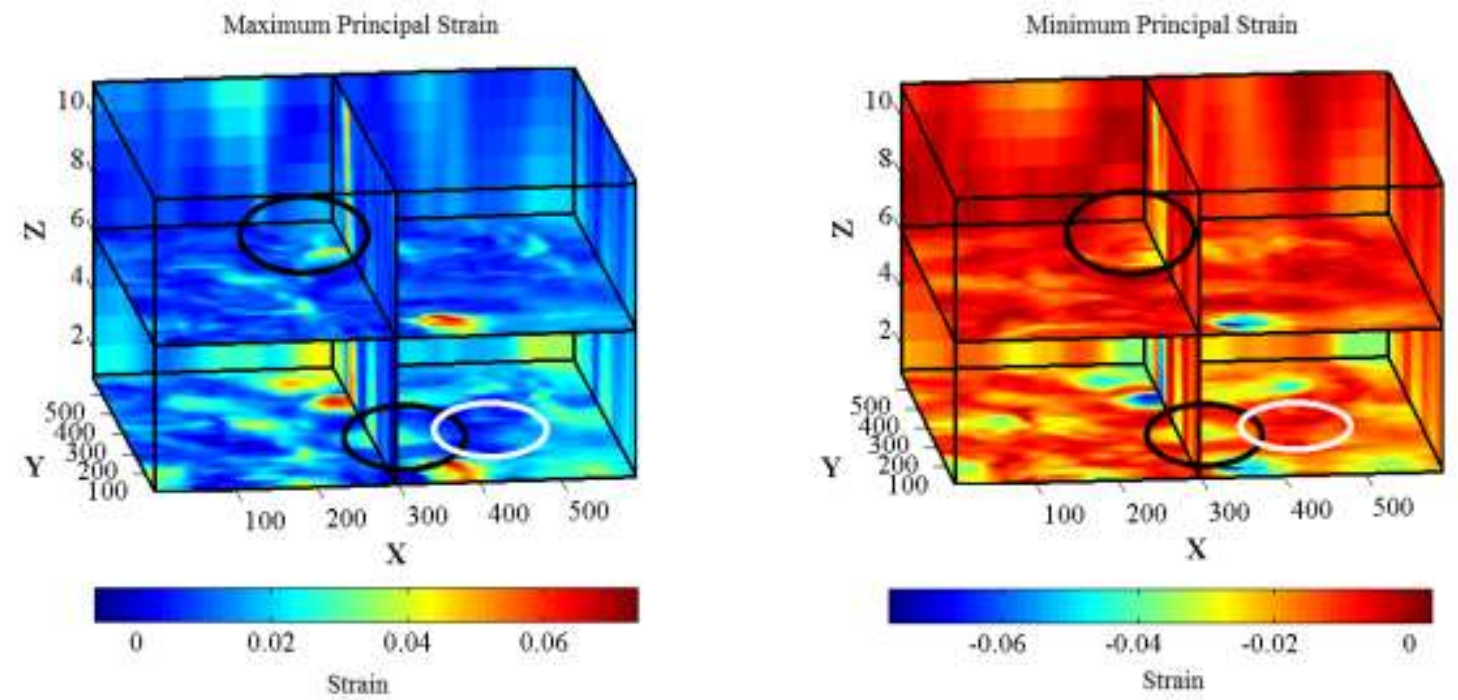

SHGM Image Volume

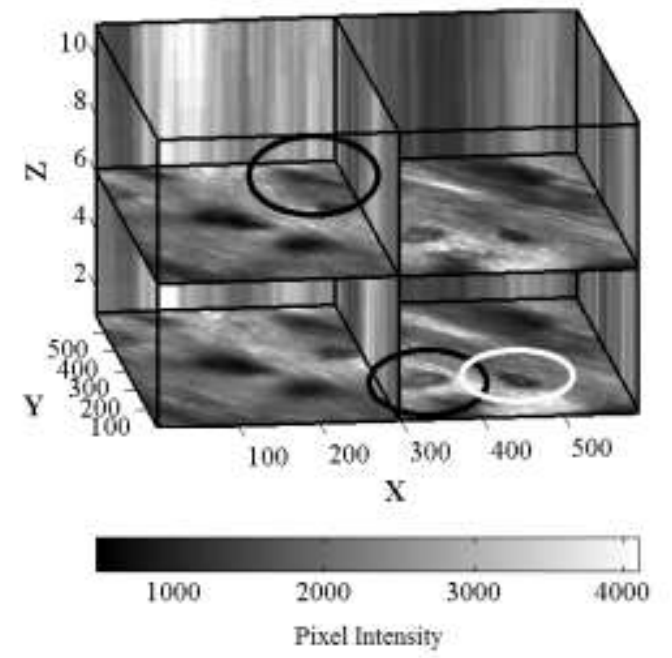

Figure 6. Three-dimensional distribution of principal strains in a representative sample. The peak maximum and minimum principal strains is shown to be localized to only a few lacunae (black ellipses), while other lacunae do not experience increased strain (white ellipses). The change in strain profile with image depth can also be seen. 


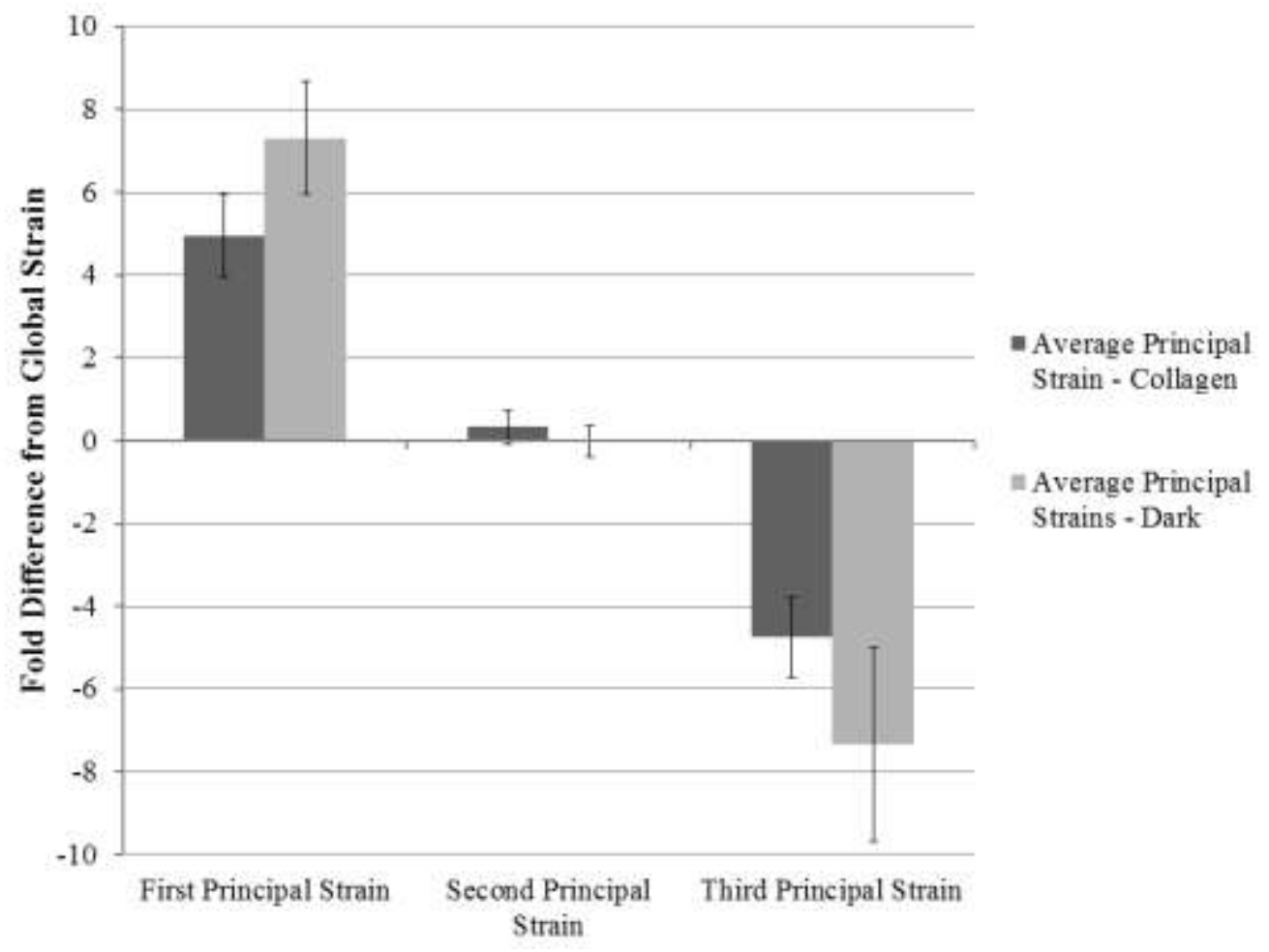

Figure 7. Comparison of principal strain magnitude in collagen bright and collagen dark regions. Collagen dark regions are expected to include both lacunae as well as some ossified bone tissue. The magnitude of both maximum and minimum principal strains was increased in collagen dark regions, but this increase was not significant. 

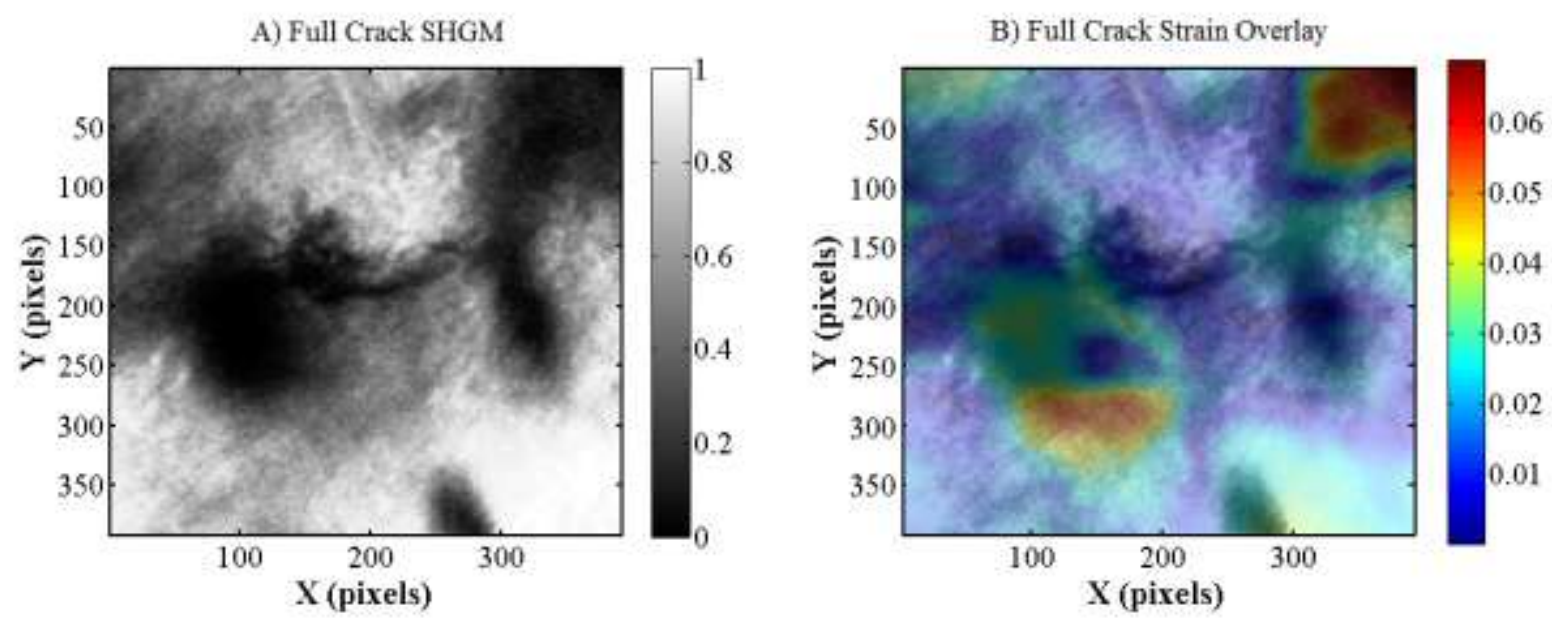

C) Crack Leading Edge SHGM
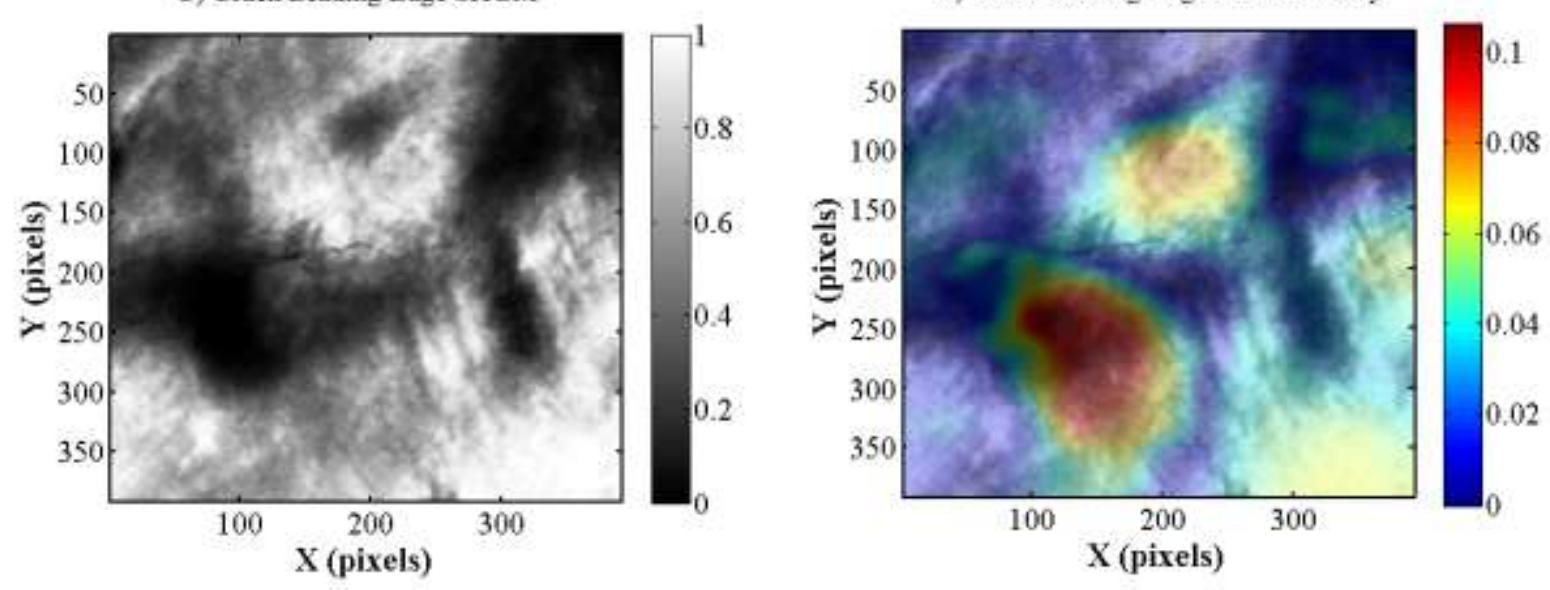

E) Before Crack Front SHGM
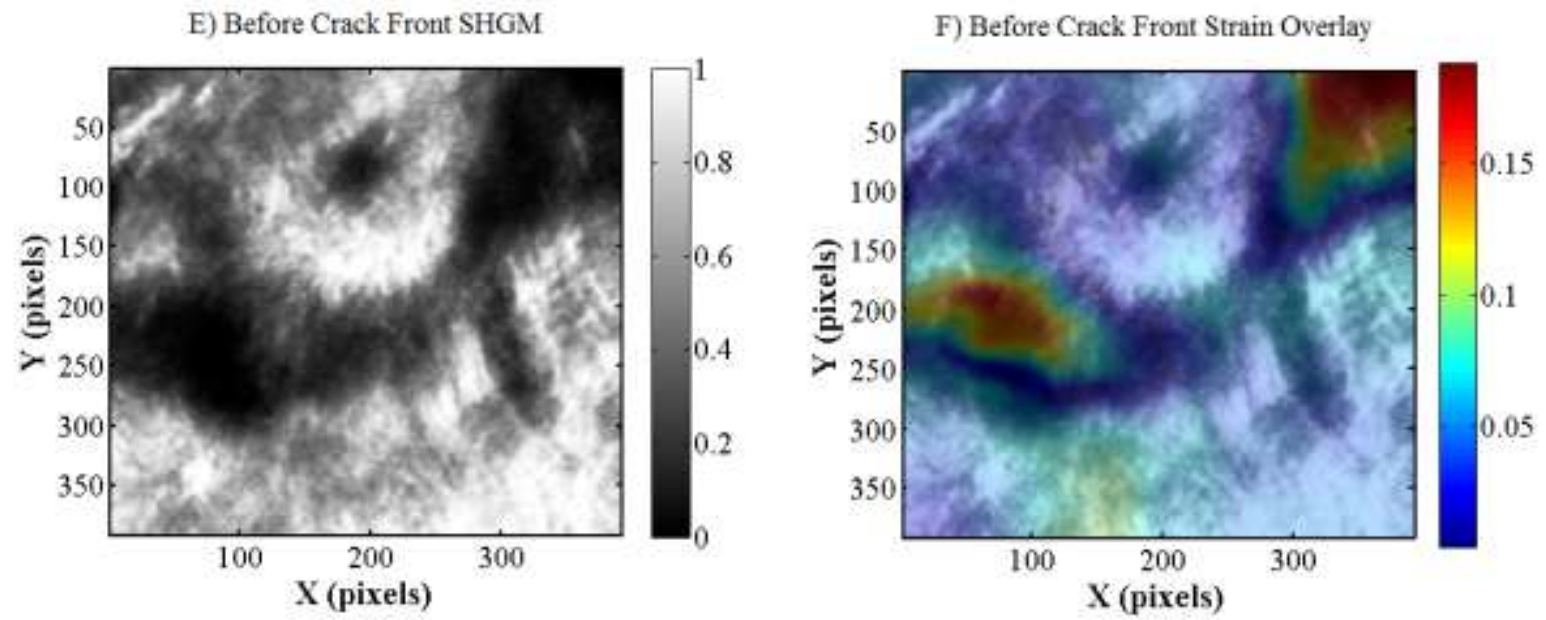

Figure 8. Tensile strains in a three dimensional analysis of a microcrack between two lacunae. At both the full crack location $(A / B)$ and healthy bone preceding the crack $(E / F)$ regions of peak strain are seen at the edges of the osteocyte lacunae. However, at the crack leading edge where the crack has yet to terminate in the opposite lacuna the peak strain is localized to the region between the crack front and the 
lacuna. This supports the hypothesis that lacuna mediate microcrack expansion and mitigate further microcrack expansion in intact bone.
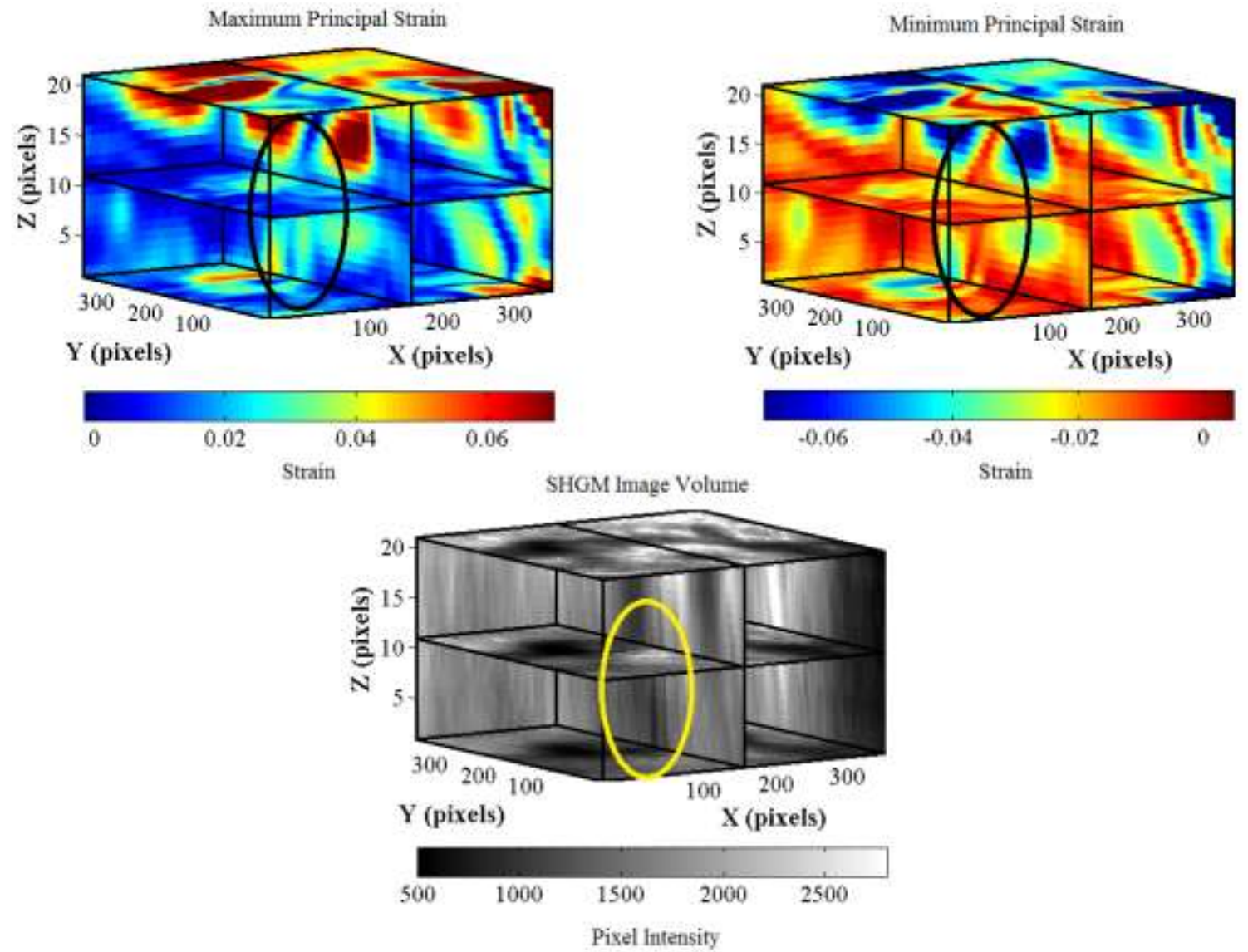

Figure 9. Three-dimensional principal strain distributions for a bone sample with a microcrack between two lacunae. The location of the crack is indicated by the ovals on each section. The location and magnitude of the strain change from the crack location to the leading edge of the crack. 